\title{
Observational Follow-Up Study of Hospitalized Patients Diagnosed with COVID-19 to Evaluate the Effectiveness of the Pharmacological Treatment Used to Treat this Disease. COVID-19 Registry
}

Lavin-Alconero $L^{1-3, *}$, Bautista-Blazquez $A^{2}$, Nogueiras-Alvarez $R^{2}$, Valencia-Lopez $D^{2}$, Cuellar-Gomez $D^{2}$, Vaca-Recalde $M G^{2}$, Alonso-Gomez B2 , Mazon-Maraña I'-3, Gonzalez-Samperio $\mathrm{C}^{1-3}$, Gimenez-Poderos $\mathrm{T}^{1,4}$, Gomez $\mathrm{D}^{4}$, Vega-Gil N ${ }^{1,2}$, and Garcia-Saiz $\mathrm{MM}^{1-3}$

${ }^{1}$ Marqués de Valdecilla Research Institute (IDIVAL), Calle Cardenal Herrera Oria, Santander, Cantabria, Spain 2Department of Clinical Pharmacology, Marqués de Valdecilla University Hospital, Santander, Cantabria, Spain ${ }^{3}$ Clinical Trial Agency, Valdecilla-IDIVAL, Marqués de Valdecilla University Hospital, Santander, Cantabria, Spain ${ }^{4}$ Hospital Pharmacia, Marqués de Valdecilla University Hospital, Santander, Cantabria, Spain

*Corresponding author: Lavin-Alconero L, Marqués de Valdecilla Research Institute (IDIVAL), Calle Cardenal Herrera Oria, Santander, Cantabria, Spain, E-mail: lucialavinalconero@gmail.com

Received: 17 Sep, 2021 | Accepted: 15 Oct, 2021 | Published: 21 Oct, 2021

Citation: Lavin-Alconero L, Bautista-Blazquez A, Nogueiras-Alvarez R, Valencia-Lopez D, Cuellar-Gomez D, et al. (2021) Observational Follow-Up Study of Hospitalized Patients Diagnosed with COVID-19 to Evaluate the Effectiveness of the Pharmacological Treatment Used to Treat this Disease. COVID-19 Registry. J Drug Res Dev 7(2): dx.doi.org/10.16966/2470-1009.166

Copyright: (c) 2021 Lavin-Alconero L, et al. This is an open-access article distributed under the terms of the Creative Commons Attribution License, which permits unrestricted use, distribution, and reproduction in any medium, provided the original author and source are credited.

\section{Abstract}

Background: During the early months of the pandemic (between March and April 2020), a pragmatic, non-commercial clinical investigation was conducted to recognize the clinical status and severity of patients hospitalized with COVID-19, in accordance with available treatments authorized by regulatory agencies.

Methods: A post-authorization observational study of COVID-19-infected patients was designed. The study is a registry-based cohort of patients.

The main objectives were to evaluate the treatment response and recovery of the included patients, and to know the causes of mortality.

A descriptive analysis of the data was performed using mean and standard deviations for quantitative variables, and frequencies for qualitative variables.

The protocol was registered in clinical trials NCT04347278 and The European Network of Centres for Pharmacoepidemiology and Pharmacovigilance EUPAS34551.

Conclusions: In the early stages of the pandemic, knowledge on effective treatments for COVID-19 patients was limited. Therefore, this study protocol aimed to combine diverse medical specialist' perspectives on the disease, record variables that would help understand the clinical evolution of COVID-19 patients and establish effective treatment strategies.

Keywords: SARS-CoV-2; Hospitalized; Quality life; Registry; Treatment

\section{Background}

The Spanish Agency of Medicines and Health Products (AEMPS) continuously monitors, together with experts from European agencies, the EMA and the rest of the world agencies, all the data on the use of drugs to treat COVID-19. This is a constantly changing scenario due to the enormous amount of data, communications and publications that have been generated worldwide. During the first wave of the pandemic, the period from March to April 2020, only partial, preliminary, sometimes only in vitro or even contradictory data on the efficacy of one or another product were available, so, when possible, priority was given to conducting clinical studies that would generate useful knowledge while offering a plausible alternative treatment.

The proposed drugs were reflected by the AEMPS and were included in the technical document "Clinical management of COVID-19: medical treatment" dated March and June of 2020, prepared by the Ministry of Health and various scientific societies [1]. The main objective of the document was the clinical management, prevention and control of the disease and, it has been amended several times:

18.05.2020: The available medical treatments were modified and definitions were adapted. 
18.06.2020: The treatment considerations for COVID-19 were corrected and the management of isolation was adapted.

In addition, to drug therapies, the following objectives within the management of hospitalized adults were taken into account to design the protocol:

\section{Initial assessment and hospital admission considerations}

a. For patients with mild symptomatology, home isolation was proposed for up to 10 days from the onset of symptoms. Clinical follow-up was supervised by their respective primary care physician.

b. The early detection of severe cases was a key to optimize individualized treatments and improve patient admission to intensive care units.

\section{Severity assessment}

Pneumonia severity was assessed based on both the ATS/IDSA and CURB65 scale.

The patient's clinical stability was assessed by their vital signs, which were surveilled and monitored every 8 hours until clinical stability was achieved.

\section{Treatment strategies}

Although there was no evidence for the effective treatment of COVID-19 infection-treatment recommendations available at the moment were recorded in table 1 .

Available treatments for the management of respiratory infection of SARS-CoV-2 (AEMPS) regarding availability and access conditions of the specific treatments used against SARS-CoV-2 infection [2-4]. In addition to the recommended treatment strategies proposed, inclusion in clinical trials as an alternative option to antiviral treatment and access to certain drugs is under compassionate use or for off-label use by signing an informed consent form was contemplated [5].

The available treatments corresponded to the medication that had been used at some point to treat COVID-19 respiratory infection. The drugs available were: Remdesivir, Lopinavir/Ritonavir, Chloroquine/ Hydroxychloroquine, Tocilizumab, Sarilumab, Ruxolitinib, Siltuximab, Baricitinib, Anakinra, Interferon Beta-1B and Interferon Alpha-2B [6].

Information regarding available treatments was amended 8 times since its first publication on March 192020 through to May 22, 2020 [1].
Due to the limited knowledge on COVID-19 therapies at the start of the pandemic, a need to establish treatment guidelines and identify prognostic clinical variables was fundamental. Consequently, this protocol was elaborated with the purpose of establishing new strategies to compliment the lack of knowledge at the time.

\section{Objectives and Purpose of the Study}

\section{Main objective}

The efficacy of pharmacological treatments used in hospitalized patients with SARS-CoV-2 infection (COVID-19 patients) in routine clinical practice, measured in terms of clinical stability, recovery and mortality during the first wave of infection was determined.

\section{Secondary objectives}

The secondary objectives studied in the protocol were the following:

i. The possible risk factors or modifiers of pharmacological effect such as demographic characteristics, comorbidities or underlying pathology and concomitant medication were identified.

ii. Information on the patterns of use of these drugs (dosage, duration of treatment, and use according to the Technical Document of the Ministry of Health) were obtained.

iii. The adverse effects of the drugs used were identified and quantified.

iv. Other treatment effectiveness data such as time of hospital admission and/or Intensive Care Unit (ICU) stay and maximum severity achieved were recorded.

v. Patients positive for SARS-CoV-2 who had not been treated with any of the drugs described in the protocol were identified and their evolution was determined.

vi. The quality of life perceived by patients after recovery/hospital discharge was assessed.

\section{Study Methodology}

\section{Study design}

Post-authorization observational study of patients with SARSCoV-2 infection (COVID-19). This study included a cohort of hospitalized and treated patients and took the form of a patient registry.

Table 1: Treatment recommendations.

\begin{tabular}{|c|c|c|c|}
\hline Symptoms & X-ray/CT thorax & Treatment & Approach \\
\hline \multirow{2}{*}{$\begin{array}{l}\text { MILD: } \\
\text { No hypoxemia, no respiratory distress }\end{array}$} & $\begin{array}{l}\text { Does not apply except for risk } \\
\text { groups }\end{array}$ & Symptomatic (1) & Discharge home except risk groups \\
\hline & $\begin{array}{l}\text { Findings compatible with } \\
\text { pneumonia }\end{array}$ & $\begin{array}{l}\text { Specific treatment against SARS- } \\
\operatorname{CoV}-2^{*}(1)\end{array}$ & Surveillance/ Household admission \\
\hline \multirow{2}{*}{$\begin{array}{l}\text { MODERATE: } \\
\text { Hypoxemia and/ or moderate } \\
\text { respiratory distress }\end{array}$} & Normal & Symptomatic (1) & $\begin{array}{l}\text { Hospital admission without } \\
\text { antiviral treatment }\end{array}$ \\
\hline & Infiltrate & $\begin{array}{l}\text { Specific treatment against SARS- } \\
\operatorname{CoV}-2^{*}(1)\end{array}$ & $\begin{array}{l}\text { Hospital admission with antiviral } \\
\text { treatment consideration }\end{array}$ \\
\hline $\begin{array}{l}\text { SEVERE (ICU): } \\
\text { Severe hypoxemia, severe respiratory } \\
\text { distress, bad appearance }\end{array}$ & Infiltrates & $\begin{array}{l}\text { Specific treatment against SARS- } \\
\operatorname{CoV}-2^{*}(1)\end{array}$ & $\begin{array}{l}\text { Hospital admission with antiviral } \\
\text { treatment combination. } \\
\text { Consider compassionate use. }\end{array}$ \\
\hline
\end{tabular}

1: Empiric antibiotic therapy when bacterial co-infection or infection is suspected. 


\section{Study population}

Study subjects were people with a COVID-19 diagnostic who had been admitted to a hospital center either as an inpatient or in the ICU during the first wave of the pandemic.

\section{Inclusion criteria}

a. Patients of any age and sex, including minors and pregnant women.

b. Patients hospitalized with a diagnosis of COVID-19 according to the clinical and microbiological criteria established by the Health Authorities and clinical practice (these have been modified based on the "Technical document. Clinical management of COVID-19: hospital care of March 2020 version" of the Ministry of Health) [1].

c. Patients who received any specific treatment to treat COVID-19 disease (according to the "Technical Document. Clinical management of COVID-19: medical treatment of March 2020 version" of the Ministry of Health, and "Available treatments for the management of SARS-CoV-2 respiratory infection" of the AEMPS) [1].

d. Patients who were admitted but did not receive specific treatment to treat COVID-19 infection.

\section{Exclusion criteria}

a. Patients who were unwilling to give informed consent upon requested.

b. Patients selected met all the inclusion criteria and none of the exclusion criteria.

c. The ethics committee was asked to waive consent for the inclusion of retrospective data. However, a verbal request was made by telephone as to whether the patient wished to continue in the study and whether he/she agreed to answer the questions in the follow-up visit questionnaire carried out between 6 months and one year after admission.

\section{Description of treatment}

The data collected on the treatments of the patients included was in accordance with the latest document of the Spanish Ministry of Health [1]. Treatments included were the following: Lopinavir/ Ritonavir; Hydroxychloroquine or Chloroquine; Interferon Beta1B or Interferon Alfa 2B; Remdesivir; Intravenous Immunoglobulin; Tocilizumab; Sarilumab; Systemic corticosteroids and lastly, Anakinra.

Study variables: The primary (response) variables were:

a. Response to treatment and time to response (from initiation of treatment).

b. Assessment of response: Adequate response to treatment meant reaching clinical stability 3-4 days after treatment assessed using the criteria set out below:

c. Recovery and time to recovery (from start of treatment)

d. Assessment of recovery: patient in whom symptoms had disappeared and had two consecutive negative PCRs separated by at least 24 hours.

e. Mortality (causes) and time to death (from start of treatment).

Exposure variables: Specific treatment for COVID-19 received during admission: drug(s), regimen/dose, start date and end date for each.
Other variables: This include(s);

- Demographic data: sex, age, race.

- The start and end date of the symptomatology presented by patients (especially fever, cough, dyspnea, malaise, myalgia/ arthralgia or other atypical symptoms).

- Diagnosis: criteria (clinical and/or microbiological) and date. The dates and results of PCR in nasopharyngeal swabs or other respiratory samples performed on each patient, or other etiological diagnostic test to be performed in the future, were collected.

- Hospitalization: severity at admission (according to the levels shown in the table 1) and length of hospitalization (days).

- ICU hospitalization: severity at admission, criteria for admission, length of stay in ICU, need for mechanical ventilation, vasopressors, ECMO and other complications.

- Basic pharmacological treatment for these diseases.

- Laboratory parameters: biochemistry, blood count, gasometry, immunochemistry; with special reference to prognostic or severity indicators such as ferritin, lactate, D-dimer, procalcitonin, IL-6.

- Microbiological parameters: in addition to those specific for SARS-CoV-2 (PCR, IgM, IgG, Ag), serology for other viruses and culture results.

- Diagnostic imaging tests: radiological pattern.

- Suspected adverse reactions to treatments received: type, date, severity, previous knowledge, and outcome.

- Quality of life questionnaire on care received during hospitalization and after recovery: SF-36.

\section{Work Plan (Table 2)}

\section{Ethical Aspects and Protection of Participating Subjects}

The study was conducted in accordance to the principles of the Declaration of Helsinki adopted by the $18^{\text {th }}$ World Medical Assembly, Helsinki, Finland in 1964 and amended in Tokyo (1975), Venice (1983), Hong Kong (1989), South Africa (1996), Edinburgh (2000), Washington (2002), Tokyo (2004) and Seoul (2008) and Brazil (2013); and the Laws and Regulations in force in Europe and Spain.

Because the study was performed under clinical practice routine conditions, it did not have any direct medical benefit or risk for the patient, since it did not involve any change in their treatment, nor in other aspects of their usual daily activities.

An informed consent waiver was been obtained for the performance of this study, although the patient's verbal consent was requested during the telephone visit made 6 months after discharge.

In order to guarantee the confidentiality of the data of the patients participating in the study, only the investigator and his team of collaborators, the representative of the sponsor who carried out the monitoring tasks, the auditor in the event that the study is submitted to an audit, the Ethics Committee and the Health Authorities had access to the data.

The processing, communication and transfer of personal data of all participating subjects will complied with the provisions of the Organic Law 3/2018 of the $5^{\text {th }}$ of December on the Protection of Personal Data and guarantee of digital rights, and the application of the Regulation (EU) 2016/679 of the European Parliament and of the Council of the $27^{\text {th }}$ April 2016 on Data Protection. 
Table 2: Work plan.

\begin{tabular}{|c|c|c|c|c|}
\hline Collection Data & $\begin{array}{c}\text { Selection } \\
\text { ( } \pm 5 \text { days after } \\
\text { diagnosis) }\end{array}$ & During hospitalization & Hospital discharge & $\begin{array}{c}\text { Follow-up visit(s) } \\
\text { (in any of the } 6 \text { months } \\
\text { after discharge) }\end{array}$ \\
\hline Signature of $\mathrm{HIP} / \mathrm{Cl}$ if applicable & $\mathrm{x}$ & & & \\
\hline Inclusion/exclusion criteria & $x$ & & & \\
\hline Demographic data and history & $\mathrm{x}$ & & & \\
\hline Clinical data: symptoms, examination, severity & $x$ & $x$ & $x$ & $x$ \\
\hline Laboratory data & $x$ & $x$ & $x$ & $x$ \\
\hline Radiological data & $\mathrm{x}$ & $x$ & $x$ & $x$ \\
\hline Treatment under study & & $x$ & $x$ & $\mathrm{x}$ \\
\hline $\begin{array}{l}\text { Evaluation of clinical evolution: stability, } \\
\text { recovery or death }\end{array}$ & & $x$ & $x$ & $x$ \\
\hline Hospital stay & & & $x$ & \\
\hline Questionnaires & & & & $x$ \\
\hline Concomitant medication & $\mathrm{x}$ & $x$ & $\mathrm{x}$ & $x$ \\
\hline Adverse events & & $x$ & $x$ & $x$ \\
\hline
\end{tabular}

During the course of the study, all documents related to the study were located in a secure area of the center. The file containing patient identifiable data was under the responsibility of the investigators and no files used for data collection or analysis carried patient identifiable data. The database generated by the study did not contain any patient identification. Thus, patients were not identifiable, since this database did not contain information on patient's initials, history numbers or any other identifying information related to these patients.

The performance of this study did not interfere with the prescribing habits of the drugs under study. These drugs were prescribed on the basis of exceptional conditions according to clinical practice.

\section{Management and Reporting of Adverse Reactions}

The study sponsor reported suspected serious unexpected adverse reactions detected in the study based on the following criteria and definitions:

\section{Adverse reaction}

Any response to a drug that is noxious and unintended, and that occurs at doses normally applied in humans for the prophylaxis, diagnosis, or treatment of disease, or for the restoration, correction, or modification of physiological functions. This term also includes all harmful clinical consequences resulting from drug dependence, abuse and misuse, including those caused by use outside authorized conditions and those caused by medication errors.

\section{Serious adverse reaction}

Any adverse reaction that results in death, may be life-threatening, requires hospitalization of the patient or prolongation of existing hospitalization, results in significant or persistent disability or incapacity, or constitutes a congenital anomaly or birth defect. For reporting purposes, suspected adverse reactions that are considered medically important even if they do not meet the above criteria such as those that put the patient at risk or require intervention to prevent any of the above outcomes will also be treated as serious. Likewise, for reporting purposes, all suspected transmission of an infectious agent through a drug will be treated as serious.

\section{Unexpected adverse reaction}

Any adverse reaction whose nature, severity or consequences are not consistent with the information described in the technical data sheet.

The notification was made to the Pharmacovigilance Center of the corresponding Autonomous Community through the usual channels for spontaneous notification by healthcare professionals (Yellow Card), which may have been:

- $\quad$ Paper notification on the usual notification form i.e., the yellow card that was sent to the aforementioned center.

- On-line notification through the web page https://www. notificaram.es/ following the instructions provided therein.

In any of the cases it was stated that the notification came from an EPA, indicating the name and code of the study from which it came from.

\section{Monitoring and Data Management}

The data collection form was REDCAP (Research Electronic Data Capture) and its system allowed data entry by project researchers. The instructions for recording the cases were in accordance with the study protocol. The SCReN platform staff that followed the monitoring plan approved for the study and ensured the quality of the data, carried out the data review or monitoring process.

\section{Confidentiality}

Personal data was processed in accordance with Regulation (EU) 2016/679 of the European Parliament and of the Council of 27 April 2016 on the protection of individuals with regards to the processing of personal data and on the free movement of such data, and the relevant local laws. The data collected for the study was identified by an alphanumeric code in such a way that it was not possible to identify the patient. Only the investigator and authorized people involved in the study had access to this code and used this information exclusively for the purposes of the study. All the generated data was recorded in the CRF in an anonymized form. 


\section{Conflicts of Interest}

We have no conflicts of interest to disclose.

\section{Acknowledgements}

This study was designed in the early days of the COVID-19 pandemic, therefore, we thank the medical staff for all their support, collaboration and effort in the development of the study, thanks to the IDI-REM-2021 team: MARIA ANGELES DE COS COSSIO (Specialist in Clinical Pharmacology), MARIA BLANCA SANCHEZ SANTIAGO (specialist in Clinical Pharmacology), MARCOS LOPEZ HOYOS (specialist in Immunology), JAVIER CRESPO GARCIA (specialist in Digestive), JOSE MANUEL OLMOS MARTINEZ (specialist in Internal Medicine), JOSE LUIS HERNANDEZ HERNANDEZ (specialist in Internal Medicine), BORJA SUBERVIOLA CAÑAS (specialist in Intensive care), CARMEN FARIÑAS ÁLVAREZ (specialist in Infection diseases) JOSE MANUEL CIFRIAN MARTÍNEZ (specialist in Pneumology), JORGE CALVO MONTES (specialist in Microbiology).

\section{References}

1. AEMPS (2020) Clinical management of COVID-19: hospital care. Ministry of Health.

2. AEMPS (2020) Treatments available for the management of respiratory infection by SARS-CoV-2. Agencia Española de Medicamentos y Productos Sanitaros.

3. World Health Organization (2020) Clinical management of severe acute respiratory infection when Novel coronavirus (2019-nCoV) infection is suspected: Interim Guidance, 28 January 2020. WHO.

4. Centers for Disease Control and Prevention (2020) Interim Infection Prevention and Control Recommendations for Healthcare Personnel during the Coronavirus Disease 2019 (COVID-19) Pandemic. CDC.

5. Lu $\mathrm{H}$ (2020) Drug treatment options for the 2019-new coronavirus (2019-nCoV). Biosci Trends 14: 69-71.

6. Ying L (2020) A randomized, open, controlled study evaluating the efficacy and safety of lopinavir/ritonavir in the treatment of hospitalized patients with 2019 novel coronavirus (COVID-19) infection. Chinese Clinical Trial Registry ChiCTR2000029308. 